\title{
Análise crítica do índice de qualidade da dieta revisado para a população brasileira (IQD-R): aplicação no ELSA-Brasil
}

\author{
Critical analysis of the revised diet quality index for the Brazilian \\ population (DQI-R): its application in ELSA-Brasil
}

\author{
Raphaela Kistenmacker Pires (https://orcid.org/0000-0003-3683-9724) ${ }^{1}$ \\ Vivian Cristine Luft (https://orcid.org/0000-0003-2371-9264) ${ }^{2}$ \\ Marina Campos Araújo (https://orcid.org/0000-0002-7980-6618) ${ }^{1}$ \\ Daniel Bandoni (https://orcid.org/0000-0003-1638-1437) ${ }^{3}$ \\ Maria del Carmen Molina (https://orcid.org/0000-0002-8614-988X) ${ }^{4}$ \\ Dora Chor (https://orcid.org/0000-0002-3941-5786) ${ }^{1}$ \\ Letícia de Oliveira Cardoso (https://orcid.org/0000-0003-1312-1808) ${ }^{5}$
}

${ }^{1}$ Escola Nacional de Saúde Pública Sérgio Arouca, Fiocruz. R. Leopoldo Bulhões 1480, Manguinhos. 21041-210 Rio de Janeiro RJ Brasil. raphaelakpires.nut

@gmail.com

${ }^{2}$ Programa de Pós-

Graduação em

Epidemiologia,

Universidade Federal do Rio

Grande do Sul. Porto Alegre

RS Brasil.

${ }^{3}$ Instituto de Saúde e Sociedade, Universidade

Federal de São Paulo. Santos SP Brasil.

${ }^{4}$ Centro de Ciências da Saúde, Universidade Federal do Espírito Santo. Vitória ES Brasil.

${ }^{5}$ Departamento de Epidemiologia e Métodos Quantitativos em Saúde,

Fiocruz. Rio de Janeiro RJ Brasil.

\begin{abstract}
The scope of this study was to conduct a critical analysis of the application of the Brazilian Healthy Eating Index - Revised (BHEI-R), to explain the ease and difficulties in its calculation, to suggest adaptations and to compare its distribution. This was done in accordance with sociodemographic variables among the 15,105 public servants participating in the Longitudinal Study of Adult Health from 2008 to 2010. Food consumption was assessed based on a Food Frequency Questionnaire and BHEI-R was estimated in four ways: original; weighted for frequency of consumption of fruits and vegetables; modified considering legumes separated from other vegetables, and adapted covering the two previous changes. The results indicated that irrespective of the adaptation performed, women, individuals over 65 years of age and individuals with lower schooling had higher mean scores indicating a better quality diet. It is believed that the proposed adaptations may be useful for future studies that apply BHEI-R.

Key words Diet, Dietary pattern, Healthy Eating Index, Adults
\end{abstract}

Resumo O objetivo deste estudo foi analisar criticamente a aplicação do Índice de Qualidade da Dieta - Revisado (IQD-R), explicitar facilidades e dificuldades em seu cálculo, sugerir adaptações e comparar sua distribuição segundo variáveis sociodemográficas entre os 15.105 servidores públicos participantes do Estudo Longitudinal de Saúde do Adulto, no período de 2008 a 2010. O consumo alimentar foi aferido com base em Questionário de Frequência Alimentar e o IQD-R foi estimado de quatro maneiras: original; ponderado para frequência de consumo de frutas e hortaliças; modificado considerando leguminosas separado dos demais vegetais e adaptado abrangendo as duas alterações anteriores. Os resultados indicaram que independentemente da adaptação realizada, as mulheres, os indivíduos com mais de 65 anos e os indivíduos de menor escolaridade apresentaram escores médios mais altos indicando dieta de melhor qualidade. Acredita-se que as adaptações propostas podem ser úteis para estudos futuros que apliquem o IQD-R.

Palavras-chave Dieta, Padrão alimentar, Índice de Qualidade da Dieta, Adultos 


\section{Introdução}

A mensuração e avaliação do consumo alimentar são procedimentos complexos no contexto clínico e no campo da pesquisa epidemiológica. Alguns índices baseados em critérios conceituais de alimentação saudável e de recomendações nutricionais têm sido propostos. Esses índices têm como objetivo a avaliação da qualidade global da dieta, visto que seus componentes geralmente são quantificados e, quando computados, oferecem uma medida sumária ${ }^{1-3}$. Sua aplicação, no entanto, demanda adaptações específicas para cada população, com intuito de reproduzir a realidade local.

No Brasil, a aplicação de índices de avaliação da dieta é relativamente recente. Fisberg et al. ${ }^{4}$, em 2004, adaptaram e validaram o índice americano Healthy Eating Index - $\mathrm{HEI}^{5}$ (considerado, pela American Dietetic Association, um instrumento adequado para medir a qualidade global da alimentação na população americana) para a população brasileira, gerando o Índice de Qualidade da Dieta (IQD). Em 2005, o HEI foi modificado (HEI-2005) ${ }^{6}$ para atender um conjunto de novas Diretrizes Dietéticas Americanas, incorporando aspectos da qualidade da dieta, como consumo de cereais integrais, tipos específicos de gordura e valor energético proveniente da ingestão de gordura sólida (saturada e trans), álcool e açúcar de adição. A partir dessa revisão do HEI e ao mesmo tempo em que o Guia Alimentar para a População Brasileira ${ }^{7}$ era elaborado em 2006, Previdelli et al. ${ }^{8}$ construíram o Índice de Qualidade da Dieta Revisado para População Brasileira (IQD-R), em 2011, que incluía doze componentes, dos quais nove são grupos de alimentos, dois são nutrientes e um representa a soma do valor energético proveniente da ingestão de gordura sólida, álcool e açúcar de adição.

Apesar de já ter sido demonstrada boa confiabilidade e validade relativa do IQD-R para avaliação e monitoramento da qualidade da dieta de brasileiros ${ }^{9}$, ao contrário do que vem sendo verificado em relação ao HEI-2005 $5^{10-13}$ este índice ainda não foi amplamente aplicado em estudos nacionais, sendo sua metodologia ainda passível de adaptações diante da variedade de itens alimentares presente na dieta de diferentes grupos populacionais.

Dessa forma, o objetivo do presente estudo foi analisar criticamente a aplicação do IQD-R, explicitar facilidades e dificuldades em seu cálculo, bem como o de seus componentes, sugerir adaptações e comparar sua distribuição segundo variáveis sociodemográficas entre participantes de estudo multicêntrico, o Estudo Longitudinal de Saúde do Adulto (ELSA-Brasil).

\section{Métodos}

\section{População de estudo}

Trata-se de uma análise transversal dos dados provenientes da linha de base do ELSA-Brasil, estudo de coorte multicêntrico com objetivo de investigar a incidência e os fatores de risco para doenças crônicas, em especial doenças cardiovasculares e o diabetes. A coleta de dados referentes à linha de base ocorreu no período de 2008 a 2010 com a participação de 15.105 adultos entre 35 e 74 anos de idade, servidores dos níveis de apoio, técnico e superior, de seis instituições públicas de pesquisa e ensino superior das regiões de 6 cidades do país.

O protocolo de pesquisa do ELSA-Brasil foi aprovado pelos comitês de ética de cada instituição e também pela Comissão Nacional de Ética em Pesquisa - CONEP (carta no 976). Maiores detalhes da amostra e coleta de dados foram previamente publicados ${ }^{14}$.

\section{Aferição do consumo alimentar}

$\mathrm{Na}$ avaliação do consumo alimentar foi utilizado o questionário de frequência alimentar (QFA) semiquantitativo validado ${ }^{15}$ que estimou a dieta habitual dos últimos doze meses. Para calcular a quantidade de energia e nutrientes de cada item alimentar de acordo com sua respectiva porção de referência foi utilizado o programa de composição nutricional Nutrition Data System for Research - NDSR, da Universidade de Minnesota (Estados Unidos) ${ }^{16}$. As frequências do consumo relatadas no QFA foram transformadas em frequência diária e as quantidades foram estimadas em gramatura com base nas medidas caseiras e/ou porção de referência de cada item alimentar. Detalhes adicionais sobre o QFA e os cálculos envolvidos para gerar informações do consumo alimentar e ingestão de nutrientes podem ser obtidas em artigo que detalha o desenvolvimento do QFA ELSA-Brasil ${ }^{15}$.

\section{Avaliação do estado nutricional}

O peso foi aferido por meio de balança digital da marca Filizola ${ }^{\circledR}$ com precisão de $100 \mathrm{~g}$ e a altura aferida com auxílio de fita métrica afixada 
em parede plana da marca SECA ${ }^{\circledR}$ com o participante em posição ortostática seguindo-se o plano de Frankfurt. Em ambos os casos os participantes vestiam uniformes padronizados para posterior ajuste das medidas de peso. $\mathrm{O}$ estado nutricional foi categorizado de acordo com o Índice de Massa Corporal em eutrófico e baixo peso (IMC $<25$ $\mathrm{kg} / \mathrm{m}^{2}$ ) sobrepeso (IMC entre 25 e $30 \mathrm{~kg} / \mathrm{m}^{2}$ ) e obesidade (IMC $>30 \mathrm{~kg} / \mathrm{m}^{2}$ ).

\section{Cálculo do IQD-R original}

Para avaliar a qualidade da dieta foi utilizado o IQD-R, uma adaptação do HEI-20056. O escore do IQD-R foi obtido pelo somatório da pontuação de doze componentes originados do HEI-2005, sendo nove referentes aos grupos de alimentos ("cereais totais", "cereais integrais", "frutas totais", "frutas integrais", "vegetais totais", "vegetais verdes-escuros e alaranjados e leguminosas", "leite e derivados", "carnes, ovos e leguminosas" e "óleos"), dois com base na ingestão de nutrientes (gordura saturada e sódio) e outro ainda referente ao valor energético proveniente da ingestão de álcool, açúcar de adição e gordura sólida, no qual este último se refere ao somatório da gordura saturada e gordura trans (Gord_AA) ${ }^{8}$.

Os itens pizza, salgados assados, salgados fritos, feijão tropeiro/feijoada, cachorro quente, estrogonofe, yakisoba, sushi e comidas baianas, presentes em especial no QFA ELSA-Brasil, por apresentarem uma variabilidade na combinação de ingredientes, dificultando o reconhecimento da contribuição de cada ingrediente para determinado grupo alimentar, não foram considerados no cômputo dos componentes referentes aos grupos de alimentos, porém nos componentes referentes a ingestão de nutrientes, tais como sódio e gorduras, essa contribuição foi computada.

Considerando a proposta original do IQD-R, os componentes "fruta integral", "vegetais verdes escuros e alaranjados e leguminosas" e "cereais integrais", que não possuíam recomendações específicas no Guia Alimentar Brasileiro de 2006, com respeito à quantidade de referência de consumo, foram atribuídos números de porções proporcionais aos componentes "frutas e suco de frutas naturais", "vegetais" e "cereais, raízes e tubérculos" presentes no guia ${ }^{8}$. Além disso, para os alimentos presentes no grupo das leguminosas, em especial os feijões que representam de forma importante o hábito alimentar do brasileiro ${ }^{17}$, o IQD-R considera o cômputo das porções desse grupo de forma diferente da proposta americana, incorporando esse grupo em seu cálculo ${ }^{8}$ dado que este item é consumido por cerca de $75 \%$ dos brasileiros ${ }^{17}$. Para o componente "sódio", também foram tomadas como base as recomendações do Guia Alimentar 2006 e para a "Gordura Saturada", foram utilizadas as diretrizes da Sociedade Brasileira de Cardiologia e as recomendações da Organização Mundial de Saúde. Não há recomendações de ingestão para a população brasileira para o componente Gord_AA. Por isso, foram adotados os pontos mínimo (0) e máximo (20) quando a ingestão era $35 \%$ e $10 \%$ do valor energético total (VET), respectivamente ${ }^{8}$, de forma semelhante aos valores adotados pelo HEI-20056 .

Quando o consumo de um determinado item era igual ou maior à porção recomendada, a pontuação total (cinco ou dez pontos de acordo com o componente alimentar analisado) foi atribuída e a ausência do consumo recebeu pontuação zero. Valores intermediários foram calculados proporcionalmente à quantidade consumida ${ }^{8}$.

O valor máximo para o IQD-R é 100 pontos. Enquanto escores elevados indicam ingestão próxima aos intervalos ou quantidades recomendadas, escores baixos indicam menor aderência às recomendações.

\section{Adaptações realizadas no cálculodo IQD-R}

Considerando que os dados de consumo alimentar foram provenientes de estimativas obtidas por QFA, que em geral está associado a um relato superestimado do consumo de frutas e hortaliças ${ }^{18}$ e, que no QFA do ELSA-Brasil, os indivíduos foram questionados sobre vários itens desse grupo de alimentos ( 15 e 16 itens relacionados ao consumo de frutas e hortaliças, respectivamente), uma ponderação foi aplicada. A ponderação baseou-se em perguntas existentes no final do questionário: "Com que frequência o (a) $\mathrm{Sr}(\mathrm{a})$ costuma comer verduras e legumes crus, cozidos ou refogados, sem incluir batatas, mandioca/aipim, inhame e cará?" e "Com que frequência o (a) $\mathrm{Sr}(\mathrm{a})$ costuma comer frutas, sem incluir sucos de frutas?"19 Assim, tentou-se minimizar possível erro de classificação ou seja, uma tendência a considerar indivíduos como "adequados" quanto ao consumo de frutas e hortaliças.

Como essas perguntas não informavam sobre a quantidade, admitiu-se para fins de ponderação que, por exemplo, o relato da frequência do consumo de frutas uma vez ao dia seria equivalente ao consumo de uma porção ao dia do grupo de frutas, como também ao relato de uma vez por semana seria igual ao consumo de $1 / 7$ porção ao dia, e assim sucessivamente. 
Para ponderação, comparou-se a quantidade em porções obtidas a partir dos itens do QFA às porções estimadas a partir das duas questões qualitativas por meio do cálculo da diferença entre o número de porções. Os indivíduos que apresentaram valores negativos, ou seja, as porções estimadas a partir do QFA foram menores do que aquelas referidas nas perguntas qualitativas atribuiu-se o valor zero, e, nenhuma ponderação foi feita. Vale observar que valores negativos foram observados em apenas 3\% e $1 \%$ dos indivíduos para o consumo de frutas e hortaliças, respectivamente. Para os demais indivíduos, em que as estimativas em porções do QFA superaram aquelas das perguntas de "ponderação", o valor encontrado dessa diferença foi subtraído do total de porções dos alimentos de cada grupo de frutas e hortaliças, gerando assim uma porção ponderada para cada grupo alimentar. Ao final, multiplicou-se a porção ponderada pela energia equivalente a cada porção de frutas e hortaliças e calculou-se a pontuação dos componentes "frutas integrais", "frutas totais" e "vegetais totais" do IQD-R. Cabe ressaltar que como não era possível distinguir os vegetais que compunham o componente "vegetais verdes-escuros e alaranjados e leguminosas" na pergunta de "ponderação", excluímos esse componente e atribuímos ao componente "vegetais totais" à pontuação máxima de dez pontos. Porém, para compararmos esse componente isoladamente nas diferentes adaptações também calculamos com a pontuação máxima de cinco pontos.

Outra adaptação realizada nesse estudo se refere à forma de estimar a contribuição das leguminosas no cálculo do IQD-R. Por sua importância consideramos a energia advinda deste grupo como um grupo à parte: "leguminosas". Desta forma, não computamos a contribuição de leguminosas junto a "carnes, ovos e leguminosas", "vegetais verdes escuros e alaranjados e leguminosas" e "vegetais totais" como na proposta original do IQD-R, uma vez que o consumo de leguminosas é significativo na população brasileira ${ }^{17} \mathrm{e}$ a contabilização deste nos demais grupos superestimaria as suas pontuações. Para criar este novo componente mantendo a estimativa original da pontuação final do IQD-R, optou-se por substituir a pontuação dos componentes "frutas totais" e "vegetais verdes-escuros e alaranjados e leguminosas", componentes estes que suas pontuações já foram consideradas nos componentes "frutas integrais" e "vegetais totais", pela pontuação do componente "leguminosas", totalizando assim para este novo componente uma pontuação máxima de 10 pontos.
Assim, construímos um IQD-R contemplando duas alterações: ponderação de frutas e hortaliças e contribuição do consumo de leguminosas como componente isolado.

Para a presente análise, os resultados do IQD-R na sua forma original foram descritos como "IQD-R original"; os resultados do cálculo do IQD-R que considerou a ponderação do consumo de frutas e hortaliças foi apresentado como "IQD-R ponderado"; aquele que levou em consideração a questão relacionada às leguminosas foi descrito como "IQD-R modificado" e, finalmente, o cálculo que levou em consideração as duas adaptações da ponderação e das leguminosas foi chamado "IQD-R adaptado". Todas essas adaptações estão esquematizadas na Figura 1.

\section{Análise dos dados}

Inicialmente foram feitas análises descritivas, por meio de medidas de tendência central (média e mediana) e dispersão (desvio-padrão, percentis e valores mínimos e máximos), para as variáveis quantitativas. Já, para as variáveis qualitativas, foram calculadas medidas de frequência absoluta e relativa.

Os doze componentes do IQD-R ("cereais totais", "cereais integrais", "frutas totais", "frutas integrais", "vegetais totais", "vegetais verdes-escuros e alaranjados e leguminosas", "leite e derivados", "carnes, ovos e leguminosas" e "óleos", "gordura saturada", "sódio" e "gord_aa") foram analisados separadamente, também por meio de análises descritivas.

O IQD-R foi comparado segundo a distribuição das seguintes variáveis: sexo, faixa etária (35|45 anos, $45 \mid-55$ anos, 55|-65 anos, $65|-| 75$ anos), nível de escolaridade (1 ${ }^{\circ}$ grau incompleto, 1 ograu completo, $2^{\circ}$ grau completo, superior completo, pós-graduação) e estado nutricional, baseado nas medidas de peso e altura e do cálculo do Índice de Massa Corporal.

A análise de significância estatística foi realizada comparando os intervalos de confiança de $95 \%$. Todas as estimativas foram calculadas utilizando o programa R 2.15.1.

\section{Resultados}

Entre os 15.105 participantes da linha de base, 256 indivíduos $(1,7 \%)$ não possuíam informações completas referentes ao questionário de frequência alimentar, condições socioeconômicas ou estado nutricional, totalizando assim 14.849 


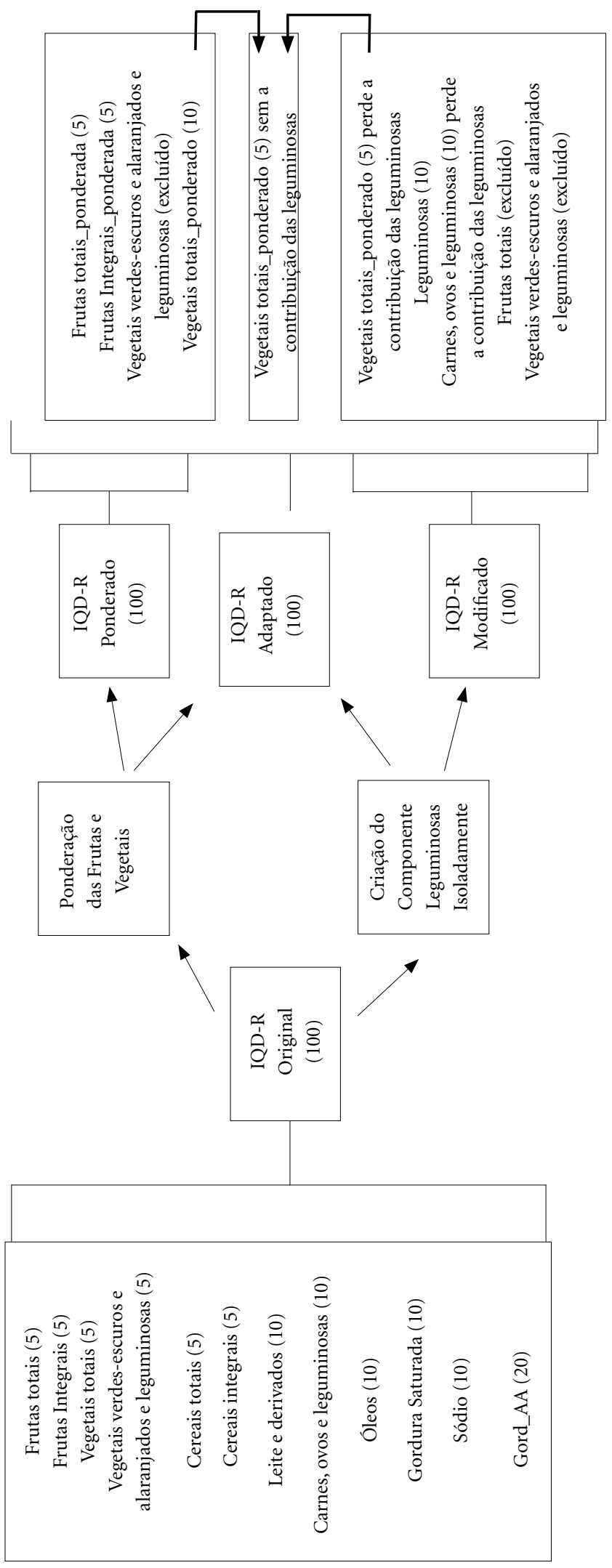

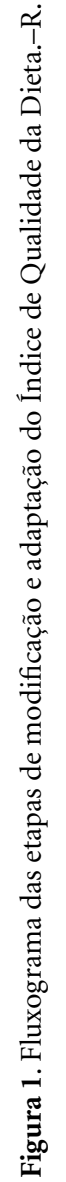


participantes. A maior parcela $(54,5 \%)$ era do sexo feminino, $36,5 \%$ apresentavam pós-graduação, aproximadamente $40 \%$ estavam na faixa etária de 45 a 55 anos e $64,5 \%$ apresentavam excesso de peso.

A média do IQD-R original em toda população foi de 72,6 pontos (pontuação máxima 100 pontos que representa quantidades recomendadas). Embora as diferenças entre as proporções dos grupos comparados de acordo com sexo, idade e escolaridade tenham sido pequenas, as mulheres, os indivíduos acima de 65 anos e aqueles de escolaridade mais baixa foram os grupos com maiores médias do escore final do IQD-R original, quando comparados às demais categorias. Não se observou diferenças estatisticamente significativas das médias do IQD-R original segundo o estado nutricional (Tabela 1).

Comparando-se a pontuação ou escore final dos respectivos IQD-R analisados, verificaram-se menores médias para o IQD-R adaptado quando comparado aos demais. Embora o comportamento do escore final em relação às variáveis independentes tenha sido semelhante nas diferentes versões do índice, a magnitude da diferença entre as médias de cada índice de acordo com as variáveis sociodemográficas foi diferente. Por exemplo, considerando-se as diferenças entre sexo, em todas as alterações do IQD-R, as mulheres apresentaram pontuações maiores do que os homens. No entanto, a diferença do escore final do IQD-R modificado foi de apenas 1,3\%, enquanto para o IQD-R ponderado foi de $4,4 \%$ (Tabela 1).

A Tabela 2 apresenta a comparação das médias da pontuação atribuída aos componentes que sofreram alterações após as adaptações empregadas no índice. A pontuação do componente "vegetais totais" foi a única que obteve valores diferentes em cada uma das versões do índice: no índice ponderado houve a ponderação do cômputo das hortaliças; no índice modificado não houve a contribuição das leguminosas, já que este foi computado como um componente a parte e no índice adaptado foi calculado considerando conjuntamente as duas alterações anteriores. Quando o IQD-R foi modificado, retirando-se a contribuição das leguminosas, foi observada discreta redução (4\%) na média da pontuação deste componente em relação ao original. Por outro lado, quando o IQD-R foi ponderado segundo o consumo de frutas e hortaliças, a média da pontuação obtida por esse mesmo componente, "vegetais totais", foi $29 \%$ menor do que a média na versão original. Além disso, o comportamento entre os sexos para esse componente no IQD-R ponderado foi diferente das demais versões. Os homens tiveram médias maiores do grupo "vegetais totais" quando comparados às mulheres. Na versão adaptada, a média deste componente foi $65 \%$ menor que a média obtida no IQD-R original, sendo esse comportamento similar de acordo com as características sociodemográficas e estado nutricional (Tabela 2).

Já para o componente "frutas integrais", verificaram-se diferenças entre as médias da pontuação do IQD-R original e ponderado. Tanto no IQD-R original quanto no ponderado, as mulheres apresentaram maiores médias de pontuação para este componente do que os homens. No entanto para o IQD-R ponderado, essa diferença entre as médias foi mais evidente e igual a 1 ponto. Observou-se também que não houve diferença entre os grupos etários para o IQD-R original, enquanto para o IQD-R ponderado, parece existir uma relação direta entre a média da pontuação desse componente e a faixa etária (Tabela 2).

A pontuação do componente "carnes e ovos" se manteve igual nos cálculos do IQD-R original e ponderado. No entanto, nas versões modificada e adaptada, este componente apresentou redução da média em aproximadamente $8 \%$ quando analisamos a população total, uma vez que o grupo das leguminosas deixou de ser considerado em sua composição (Tabela 2).

O componente "leguminosas" apresentou pontuações médias maiores entre os homens quando comparados às mulheres. Especificamente para este componente, observou-se relação inversa entre a pontuação obtida e nível de escolaridade e faixa etária. Cabe notar que a diferença entre as categorias extremas de escolaridade foi de $27,5 \%$, tendo os indivíduos de menor escolaridade a maior pontuação (Tabela 2).

Cabe notar ainda que as médias da pontuação dos componentes que sofreram alterações na versão do IQD-R original foram bem próximas à pontuação máxima ( 5 ou 10 pontos) em todas as comparações realizadas. Por outro lado, esses valores diminuíram quando as adaptações foram implementadas (Tabela 2).

\section{Discussão}

Os resultados desse estudo indicaram que independentemente da adaptação realizada, as mulheres, os indivíduos com mais de 65 anos e aqueles de menor escolaridade se aproximaram mais da dieta de melhor qualidade, ainda que a 
Tabela 1. Média e intervalo de 95\% de confiança do escore final do IQD-R original, ponderado, modificado e adaptado (pontuação máxima $=100$ ) segundo características sociodemográficas e estado nutricional. ELSABrasil, 2008-2010.

\begin{tabular}{|c|c|c|c|c|}
\hline & $\begin{array}{c}\text { IQD-R } \\
\text { Original } \\
\end{array}$ & $\begin{array}{c}\text { IQD-R } \\
\text { Ponderado }\end{array}$ & $\begin{array}{c}\text { IQD-R } \\
\text { Modificado } \\
\end{array}$ & $\begin{array}{c}\text { IQD-R } \\
\text { Adaptado }\end{array}$ \\
\hline Total & $\begin{array}{r}72,6 \\
(72,5-72,7)\end{array}$ & $\begin{array}{r}66,9 \\
(66,7-67,0)\end{array}$ & $\begin{array}{r}69,0 \\
(68,8-69,1)\end{array}$ & $\begin{array}{r}64,3 \\
(64,1-64,4)\end{array}$ \\
\hline \multicolumn{5}{|l|}{ Sexo } \\
\hline Feminino & $\begin{array}{r}73,6 \\
(73,4-73,8)\end{array}$ & $\begin{array}{r}68,2 \\
(68,0-68,4)\end{array}$ & $\begin{array}{r}69,4 \\
(69,2-69,6)\end{array}$ & $\begin{array}{r}65,2 \\
(65,0-65,4)\end{array}$ \\
\hline Masculino & $\begin{array}{r}71,4 \\
(71,2-71,6)\end{array}$ & $\begin{array}{r}65,2 \\
(65,0-65,4)\end{array}$ & $\begin{array}{r}68,5 \\
(68,3-68,7)\end{array}$ & $\begin{array}{r}63,2 \\
(63,0-63,4)\end{array}$ \\
\hline \multicolumn{5}{|l|}{ Faixa etária } \\
\hline $35-45$ & $\begin{array}{r}70,6 \\
(70,4-70,9)\end{array}$ & $\begin{array}{r}64,7 \\
(64,4-65,1)\end{array}$ & $\begin{array}{r}67,5 \\
(67,2-67,8)\end{array}$ & $\begin{array}{r}62,5 \\
(62,2-62,8)\end{array}$ \\
\hline $45-55$ & $\begin{array}{r}72,1 \\
(71,9-72,3)\end{array}$ & $\begin{array}{r}66,3 \\
(66,0-66,5)\end{array}$ & $\begin{array}{r}68,7 \\
(68,5-68,9)\end{array}$ & $\begin{array}{r}63,8 \\
(63,6-64,0)\end{array}$ \\
\hline $55-65$ & $\begin{array}{r}74,0 \\
(73,8-74,3)\end{array}$ & $\begin{array}{r}68,6 \\
(68,3-68,8)\end{array}$ & $\begin{array}{r}70,1 \\
(69,9-70,3)\end{array}$ & $\begin{array}{r}65,7 \\
(65,4-65,9)\end{array}$ \\
\hline $65-75$ & $\begin{array}{r}74,6 \\
(74,2-75,0)\end{array}$ & $\begin{array}{r}69,1 \\
(68,7-69,6)\end{array}$ & $\begin{array}{r}70,2 \\
(69,8-70,6)\end{array}$ & $\begin{array}{r}66,0 \\
(65,6-66,4)\end{array}$ \\
\hline \multicolumn{5}{|l|}{ Nível de escolaridade } \\
\hline $1^{\circ}$ grau incompleto & $\begin{array}{r}73,6 \\
(73,1-74,1)\end{array}$ & $\begin{array}{r}67,5 \\
(66,9-68,1)\end{array}$ & $\begin{array}{r}71,1 \\
(70,6-71,5)\end{array}$ & $\begin{array}{r}65,6 \\
(65,1-66,1)\end{array}$ \\
\hline $1^{\circ}$ grau completo & $\begin{array}{r}73,2 \\
(72,8-73,6)\end{array}$ & $\begin{array}{r}66,8 \\
(66,3-67,4)\end{array}$ & $\begin{array}{r}70,4 \\
(69,9-70,8)\end{array}$ & $\begin{array}{r}64,9 \\
(64,4-65,4)\end{array}$ \\
\hline $2^{\circ}$ grau completo & $\begin{array}{r}72,7 \\
(72,5-72,9)\end{array}$ & $\begin{array}{r}66,5 \\
(66,3-66,8)\end{array}$ & $\begin{array}{r}69,6 \\
(69,3-69,8)\end{array}$ & $\begin{array}{r}64,4 \\
(64,2-64,6)\end{array}$ \\
\hline Universitário completo & $\begin{array}{r}72,6 \\
(72,3-72,9)\end{array}$ & $\begin{array}{r}66,8 \\
(66,4-67,1)\end{array}$ & $\begin{array}{r}68,8 \\
(68,5-69,2)\end{array}$ & $\begin{array}{r}64,2 \\
(63,8-64,5)\end{array}$ \\
\hline Pós-graduação & $\begin{array}{r}72,2 \\
(72,0-72,4)\end{array}$ & $\begin{array}{r}67,1 \\
(66,9-67,4)\end{array}$ & $\begin{array}{r}67,9 \\
(67,7-68,1)\end{array}$ & $\begin{array}{r}63,9 \\
(63,6-64,1)\end{array}$ \\
\hline \multicolumn{5}{|l|}{ Estado Nutricional } \\
\hline Eutrófico & $\begin{array}{r}72,8 \\
(72,6-73,0)\end{array}$ & $\begin{array}{r}67,1 \\
(66,8-67,3)\end{array}$ & $\begin{array}{r}69,2 \\
(69,0-69,4)\end{array}$ & $\begin{array}{r}64,6 \\
(64,4-64,8)\end{array}$ \\
\hline Sobrepeso & $\begin{array}{r}72,5 \\
(72,3-72,7)\end{array}$ & $\begin{array}{r}66,7 \\
(66,5-66,9)\end{array}$ & $\begin{array}{r}68,9 \\
(68,7-69,1)\end{array}$ & $\begin{array}{r}64,1 \\
(63,9-64,3)\end{array}$ \\
\hline Obeso & $\begin{array}{r}72,5 \\
(72,3-72,8)\end{array}$ & $\begin{array}{r}66,8 \\
(66,5-67,1)\end{array}$ & $\begin{array}{r}68,8 \\
(68,6-69,1)\end{array}$ & $\begin{array}{r}64,1 \\
(63,8-64,4)\end{array}$ \\
\hline
\end{tabular}

pontuação tenha sido diferente de acordo com cada adaptação empregada.

Até onde é possível conhecer, essa investigação foi a primeira a verificar de forma detalhada a aplicação do IQD-R em uma grande amostra de indivíduos de seis cidades brasileiras, além de propor adaptações no cálculo do mesmo. Acreditamos que a utilização do IQD-R apresenta vantagens ao analisar sumariamente vários componentes da dieta com base na densidade energética, facilitando a mensuração de possíveis associações com desfechos de saúde ainda que seu cálculo necessite ser aperfeiçoado.
As maiores médias da pontuação do índice nas faixas etárias mais velhas podem ser explicadas pela maior preocupação com a saúde e maior ocorrência de doenças influenciando positivamente a qualidade da dieta. Além disso, a ocorrência mais frequente de doenças nessa faixa etária pode levar à modificação de hábitos, incluindo os alimentares ${ }^{20}$.

Quanto ao sexo, a maior média do escore final entre as mulheres quando comparadas aos homens se deve em grande parte à maior pontuação nos componentes cereais integrais (2,3 para mulheres e 1,7 para os homens), leite e derivados 
Tabela 2. Média e intervalo de $95 \%$ de confiança dos componentes que sofreram modificações no cálculo do IQD-R nos índices calculados, segundo características sociodemográficas e estado nutricional. ELSA-Brasil, 2008-2010.

\begin{tabular}{|c|c|c|c|c|c|c|c|c|c|}
\hline \multicolumn{10}{|c|}{ Componentes do IQD-R } \\
\hline & \multicolumn{4}{|c|}{$\begin{array}{c}\text { Vegetais Totais } \\
(\text { Pontuação Máxima }=5)\end{array}$} & \multicolumn{2}{|c|}{$\begin{array}{c}\text { Frutas Integrais } \\
\text { (Pontuação } \\
\text { Máxima = 5) }\end{array}$} & \multicolumn{2}{|c|}{$\begin{array}{l}\text { Carnes, Ovos e } \\
\text { Leguminosas } \\
\text { (Pontuação } \\
\text { Máxima = 10) }\end{array}$} & \multirow{2}{*}{$\begin{array}{c}\begin{array}{c}\text { Leguminosas } \\
(\text { Pontuação }\end{array} \\
\text { Máxima }=10) \\
\text { IQD-R } \\
\text { Mod. e Adap. }\end{array}$} \\
\hline & $\begin{array}{l}\text { IQD-R } \\
\text { Orig.* }^{*}\end{array}$ & $\begin{array}{l}\text { IQD-R } \\
\text { Pond.* }\end{array}$ & $\begin{array}{l}\text { IQD-R } \\
\text { Mod.* }\end{array}$ & $\begin{array}{l}\text { IQD-R } \\
\text { Adap.* }\end{array}$ & $\begin{array}{l}\text { IQD-R } \\
\text { Orig.e } \\
\text { Mod. }\end{array}$ & $\begin{array}{l}\text { IQD-R } \\
\text { Pond. e } \\
\text { Adap. }\end{array}$ & $\begin{array}{l}\text { IQD-R } \\
\text { Orig. e } \\
\text { Pond. }\end{array}$ & $\begin{array}{l}\text { IQD-R } \\
\text { Mod. e } \\
\text { Adap. }\end{array}$ & \\
\hline \multirow[t]{2}{*}{ Total } & 4,9 & 3,5 & 4,7 & 1,7 & 4,7 & 3,0 & 9,4 & 8,6 & 6,6 \\
\hline & $(4,9-4,9)$ & $(3,5-3,6)$ & $(4,7-4,7)$ & $(1,7-1,7)$ & $(4,7-4,8)$ & $(3,0-3,0)$ & $(9,4-9,4)$ & $(8,5-8,6)$ & $(6,6-6,7)$ \\
\hline \multicolumn{10}{|l|}{ Sexo } \\
\hline \multirow[t]{2}{*}{ Feminino } & 4,9 & 3,4 & 4,8 & 2,0 & 4,8 & 3,5 & 9,3 & 8,5 & 6,0 \\
\hline & $(4,9-4,9)$ & $(3,4-3,5)$ & $(4,8-4,8)$ & $(2,0-2,0)$ & $(4,8-4,9)$ & $(3,4-3,5)$ & $(9,3-9,3)$ & $(8,4-8,5)$ & $(6,0-6,1)$ \\
\hline \multirow[t]{2}{*}{ Masculino } & 4,8 & 3,7 & 4,5 & 1,4 & 4,6 & 2,5 & 9,6 & 8,6 & 7,4 \\
\hline & $(4,8-4,9)$ & $(3,6-3,7)$ & $(4,5-4,6)$ & $(1,4-1,4)$ & $(4,6-4,7)$ & $(2,4-2,5)$ & $(9,6-9,6)$ & $(8,6-8,7)$ & $(7,3-7,5)$ \\
\hline \multicolumn{10}{|l|}{ Idade } \\
\hline \multirow[t]{2}{*}{$35-45$} & 4,8 & 3,5 & 4,6 & 1,6 & 4,6 & 2,6 & 9,5 & 8,6 & 6,9 \\
\hline & $(4,9-4,9)$ & $(3,5-3,6)$ & $(4,5-4,6)$ & $(1,5-1,6)$ & $(4,6-4,6)$ & $(2,6-2,7)$ & $(9,5-9,6)$ & $(8,6-8,7)$ & $(6,8-7,0)$ \\
\hline \multirow[t]{2}{*}{$45-55$} & 4,9 & 3,6 & 4,7 & 1,6 & 4,7 & 2,8 & 9,5 & 8,6 & 6,8 \\
\hline & $(4,9-4,9)$ & $(3,5-3,6)$ & $(4,7-4,7)$ & $(1,6-1,7)$ & $(4,7-4,7)$ & $(2,8-2,9)$ & $(9,5-9,5)$ & $(8,6-8,7)$ & $(6,7-6,9)$ \\
\hline \multirow[t]{2}{*}{$55-65$} & 4,9 & 3,5 & 4,8 & 1,9 & 4,9 & 3,3 & 9,4 & 8,5 & 6,5 \\
\hline & $(4,9-4,9)$ & $(3,5-3,6)$ & $(4,7-4,8)$ & $(1,8-1,9)$ & $(4,8-4,9)$ & $(3,3-3,4)$ & $(9,3-9,4)$ & $(8,4-8,5)$ & $(6,3-6,6)$ \\
\hline \multirow[t]{2}{*}{$65-75$} & 4,9 & 3,3 & 4,8 & 1,9 & 4,9 & 3,6 & 9,2 & 8,3 & 6,0 \\
\hline & $(4,9-4,9)$ & $(3,2-3,4)$ & $(4,8-4,8)$ & $(1,8-2,0)$ & $(4,9-4,9)$ & $(3,5-3,7)$ & $(9,1-9,3)$ & $(8,2-8,4)$ & $(5,8-6,2)$ \\
\hline \multicolumn{10}{|l|}{ Escolaridade } \\
\hline \multirow{2}{*}{$\begin{array}{l}\text { 1.grau } \\
\text { incompleto }\end{array}$} & 4,9 & 3,7 & 4,7 & 1,4 & 4,7 & 2,5 & 9,5 & 8,3 & 8,0 \\
\hline & $(4,9-4,9)$ & $(3,6-3,8)$ & $(4,6-4,7)$ & $(1,3-1,5)$ & $(4,7-4,8)$ & $(2,4-2,7)$ & $(9,4-9,6)$ & $(8,2-8,5)$ & $(7,8-8,2)$ \\
\hline \multirow{2}{*}{$\begin{array}{l}1^{\circ} \text { grau } \\
\text { completo }\end{array}$} & 4,8 & 3,6 & 4,6 & 1,3 & 4,8 & 2,5 & 9,5 & 8,5 & 7,6 \\
\hline & $(4,8-4,9)$ & $(3,5-3,7)$ & $(4,6-4,7)$ & $(1,3-1,4)$ & $(4,7-4,8)$ & $(2,4-2,7)$ & $(9,5-9,6)$ & $(8,4-8,7)$ & $(7,4-7,8)$ \\
\hline \multirow{2}{*}{$\begin{array}{l}2^{\circ} \mathrm{grau} \\
\text { completo }\end{array}$} & 4,9 & 3,5 & 4,6 & 1,5 & 4,7 & 2,7 & 9,5 & 8,5 & 7,2 \\
\hline & $(4,9-4,9)$ & $(3,5-3,6)$ & $(4,6-4,7)$ & $(1,5-1,5)$ & $(4,7-4,7)$ & $(2,7-2,7)$ & $(9,4-9,5)$ & $(8,5-8,6)$ & $(7,1-7,3)$ \\
\hline \multirow{2}{*}{$\begin{array}{l}\text { Universitário } \\
\text { completo }\end{array}$} & 4,9 & 3,4 & 4,7 & 1,7 & 4,7 & 3,0 & 9,4 & 8,5 & 6,5 \\
\hline & $(4,9-4,9)$ & $(3,4-3,5)$ & $(4,7-4,7)$ & $(1,7-1,8)$ & $(4,7-4,8)$ & $(3,0-3,1)$ & $(9,3-9,4)$ & $(8,4-8,6)$ & $(6,3-6,6)$ \\
\hline \multirow{2}{*}{$\begin{array}{l}\text { Pós- } \\
\text { graduação }\end{array}$} & 4,9 & 3,5 & 4,7 & 2,0 & 4,8 & 3,4 & 9,4 & 8,6 & 5,8 \\
\hline & $(4,9-4,9)$ & $(3,5-3,6)$ & $(4,7-4,8)$ & $(2,0-2,1)$ & $(4,7-4,8)$ & $(3,4-3,5)$ & $(9,3-9,4)$ & $(8,6-8,7)$ & $(5,8-5,9)$ \\
\hline \multicolumn{10}{|l|}{ IMC } \\
\hline \multirow[t]{2}{*}{ Eutrófico } & 4,9 & 3,5 & 4,7 & 1,8 & 4,7 & 3,0 & 9,3 & 8,3 & 6,8 \\
\hline & $(4,9-4,9)$ & $(3,5-3,5)$ & $(4,7-4,7)$ & $(1,7-1,8)$ & $(4,7-4,8)$ & $(3,0-3,1)$ & $(9,3-9,4)$ & $(8,2-8,4)$ & $(6,7-6,9)$ \\
\hline \multirow[t]{2}{*}{ Sobrepeso } & 4,9 & 3,5 & 4,7 & 1,7 & 4,7 & 3,0 & 9,5 & 8,7 & 6,6 \\
\hline & $(4,9-4,9)$ & $(3,5-3,6)$ & $(4,7-4,7)$ & $(1,6-1,7)$ & $(4,7-4,8)$ & $(2,9-3,0)$ & $(9,4-9,5)$ & $(8,6-8,7)$ & $(6,5-6,7)$ \\
\hline \multirow[t]{2}{*}{ Obeso } & 4,9 & 3,5 & 4,7 & 1,7 & 4,7 & 3,0 & 9,5 & 8,8 & 6,4 \\
\hline & $(4,9-4,9)$ & $(3,5-3,6)$ & $(4,7-4,7)$ & $(1,6-1,7)$ & $(4,7-4,8)$ & $(2,9-3,1)$ & $(9,5-9,5)$ & $(8,7-8,8)$ & $(6,3-6,5)$ \\
\hline
\end{tabular}


(5,7 e 4,4 para mulheres e homens, respectivamente) e Gord_AA (12,3 e 11,6 para mulheres e homens, respectivamente). Esse resultado é similar ao observado na literatura ${ }^{21}$, no qual as mulheres apresentaram comportamento alimentar mais saudável quando comparadas aos homens.

Ao contrário do esperado, a pontuação final apresentou uma relação inversa com o nível de escolaridade $^{22}$. As modificações no cômputo do IQD-R ajudaram a demonstrar possíveis explicações de tal relação. Por exemplo, quando o IQD-R foi modificado acrescentando o componente "leguminosas”, notou-se que este componente apresentou grande diferença na média de pontuação entre os níveis de escolaridade, com participantes de níveis mais altos apresentando menores pontuações. Ao examinar os resultados do Inquérito Nacional de Alimentação 2008-2009 com amostra representativa da população brasileira verificou-se o papel da renda na alimentação, outro marcador de posição socioeconômica na discriminação do consumo alimentar dos brasileiros. Observou-se nesse inquérito que os indivíduos de classes de renda mais baixas consomem diversos itens considerados integrantes de uma alimentação saudável, tais como arroz e feijão, batatadoce, farinha de mandioca e milho, em maiores quantidades quando comparados aos indivíduos de classes de maior renda ${ }^{17}$. Observamos também em nossos achados esse padrão de maior consumo de itens alimentares do componente leguminosas entre indivíduos de menor escolaridade. Em contrapartida, o inquérito nacional mostrou que o consumo de frutas e verduras assim como o de leite desnatado e os derivados de leite foi maior entre brasileiros nos maiores níveis de renda ${ }^{17}$. Na mesma direção, também se verificou no presente estudo, uma pontuação média menor no componente leite e derivados entre aqueles de menor escolaridade quando comparados aos indivíduos de maior escolaridade (3,9 x 5,7, respectivamente). Entretanto, cabe mencionar que estudos adicionais são necessários para compreender melhor a relação entre o IQD-R e o nível de escolaridade no contexto brasileiro.

É relevante mencionar que o uso de diferentes métodos para aferir o consumo alimentar pode gerar resultados diferentes no índice. As diferenças encontradas nas médias das pontuações do IQD-R com uso do QFA e do R24h confirmam que o uso do QFA para aferição do consumo alimentar pode superestimar a média dos índices, dado que quando os dados são coletados por meio de QFA os escores finais do índice apresentam-se sistematicamente maiores do que aqueles coletados por meio de $\mathrm{R} 24 \mathrm{~h}^{23,24}$. O estudo de reprodutibilidade e validade relativa do QFA ELSA -Brasil ${ }^{19}$, utilizado no presente estudo, mostrou média de ingestão de energia e nutrientes maior quando comparada à média de ingestão obtida pela aplicação de três dias de registro alimentar e atribuiu à superestimativa da ingestão a ampla lista de alimentos adotada no ELSA-Brasil. Vale ressaltar que todos os métodos de aferição do consumo alimentar apresentam limitações, e a escolha do QFA ELSA-Brasil baseou-se na suposição de que ele é um método simples de se analisar, tem baixo custo e principalmente estima diretamente o consumo habitual de alimentos minimizando os erros ocasionados pela variabilidade intraindividual ${ }^{19}$.

Embora resultados relativamente consistentes com a literatura tenham sido observados, alguns aspectos metodológicos referentes ao cálculo do IQD-R são trabalhosos. A respeito do cálculo, o reconhecimento de uma receita ou ficha técnica "padrão" para preparações elaboradas, tais como pizza e comidas baianas, presentes em especial no QFA ELSA-Brasil, que podem ter uma variabilidade na combinação e gramatura de ingredientes, foi uma dificuldade importante. Nesses casos, optou-se por desconsiderar esses 11 itens no cômputo dos grupos de alimentos. Além disso, foi importante separar as leguminosas que compuseram um grupo isolado, devido à importância desse grupo de alimentos no padrão alimentar brasileiro ${ }^{17}$. Outra limitação do cômputo do índice diz respeito à pontuação máxima que os itens alimentares podem receber, de acordo com seus respectivos grupos, uma vez que indivíduos que consomem 5 porções diárias de frutas e hortaliças recebem a mesma pontuação que aqueles que consomem 10 porções. O mesmo acontece com outros grupos de alimentos, a exemplo das "carnes" e "cereais" em que indivíduos com um consumo excessivamente alto, 15 porções, recebem a mesma pontuação de indivíduos que consomem 5 porções ao dia.

Cabe salientar que embora a elaboração do IQD-R original tenha se baseado no antigo guia alimentar, publicado em $2006^{7}$, não acreditamos que o mesmo esteja em desacordo com as diretrizes do guia atual ${ }^{25}$. Isso porque este último integra uma nova classificação de alimentos baseada no grau de processamento dos alimentos, valorizando o consumo de alimentos in natura ou minimamente processados que comparativamente iriam pontuar positivamente no índice, a exemplo dos componentes frutas e hortaliças, em detrimento dos alimentos ultraprocessados, ricos em nu- 
trientes pertencentes ao componente Gord_AA e sódio, que pontuam negativamente o índice. Contudo, vale ressaltar dois aspectos importantes na abordagem do guia atual que necessitaria repensar o IQD-R. Primeiro, que o guia não recomenda porções específicas para o consumo dos referidos grupos, o que exigiria uma nova proposta de cálculo do índice. Segundo, que as recomendações do guia vão além de recomendações baseadas na adequação da ingestão de nutrientes introduzindo implicações sociais, culturais, econômicas e ambientais das escolhas alimentares, ou seja, um novo olhar sobre como os indivíduos têm se alimentado e a sustentabilidade do sistema alimentar. Esses aspectos citados anteriormente são difíceis de serem incorporados no cálculo de um índice.

O que tem sido feito internacionalmente são alterações e atualizações dos índices. O exemplo americano é o HEI-2005 que também sofreu alterações e em sua nova versão (HEI-2010) ${ }^{26}$ algumas das adequações que sugerimos no presente estudo foram adotadas, tais como a separação das leguminosas do grupo de vegetais e carnes. O HEI-2010 também criou um componente de moderação para avaliar o consumo excessivo de grãos refinados, outro ponto que discutimos anteriormente, além de separar o componente de proteínas totais, em dois grupos, aquelas de origem vegetal e as advindas de frutos do mar. A substituição do componente "óleo", que em nosso estudo recebeu a nota máxima em todas as versões, por um componente que avalia a proporção da ingestão de gordura poliinsaturada e monoinsaturada com as saturadas também parece ser vantajosa.

Conclui-se que a aplicação do índice com base em QFA pode superestimar o consumo de frutas e consequentemente a qualidade da dieta, necessitando adaptação aqui apresentada. Além disso, o consumo de feijão é um hábito bastante frequente na população brasileira e se não considerado separadamente pode superestimar o consumo de vegetais. Destaca-se ainda o desafio de incorporar no cálculo do índice as questões colocadas no guia atual brasileiro. Por fim, a coorte ELSA-Brasil apresenta uma oportunidade para o desenvolvimento de outros estudos com o objetivo de investigar a capacidade preditiva dos índices e sua associação com desfechos de saúde.

\section{Colaboradores}

RK Pires: Redigiu o manuscrito, realizou as análises de dados e contribuiu na definição do escopo do artigo. VC Luft: Revisou a análise de dados, auxiliou na interpretação dos resultados e revisou a redação do artigo. MC Araújo: propôs a definição do escopo do artigo, auxiliou na interpretação dos resultados e revisou a redação final do manuscrito. D Chor: auxiliou na formulação da pergunta de pesquisa, auxiliou na interpretação dos resultados e revisou a redação do artigo. MC Molina: auxiliou na interpretação dos resultados e revisou a redação do artigo. D Bandoni: participou na definição da metodologia adotada, auxiliou na interpretação dos resultados e revisou a redação final do artigo. LO Cardoso: Elaborou a pergunta de pesquisa, revisou a metodologia e análise implementadas, auxiliou na interpretação dos resultados, revisou a redação do artigo. 


\section{Referências}

1. Wirfält E, Drake I, Wallström P. What do review papers conclude about food and dietary patterns? Food Nutr Res 2013; 57.

2. Newby PK, Tucker KL. Empirically derived eating patterns using factor or cluster analysis: a review. Nutr Rev 2004; 62(5):177-203.

3. Patterson RE, Haines PS, Popkin BM. Diet quality index: capturing a multidimensional behavior. $J \mathrm{Am}$ Diet Assoc 1994; 94(1):57-64.

4. Fisberg RM, Slater B, Barros RR, Lima FD, Cesar CL, Carandina L, Barros MBA, Goldbaum M. Índice de Qualidade da Dieta: avaliação da adaptação e aplicabilidade. Rev Nutr 2004; 17(3):301-318.

5. Kennedy EI, Ohls J, Carlson S, Fleming K. The healthy eating index: design and applications. J Am Diet Assoc 1995; 95(10):1103-1108.

6. Guenther PM, Reedy J, Krebs-Smith SM, Reeve BB, Basiotis PP. Development and evaluation of the healthy eating index-2005. Washington: Center for Nutrition Policy and Promotion; 2007.

7. Brasil. Ministério da Saúde (MS). Guia alimentar para a população brasileira: promovendo a alimentação saudável. Brasília: MS; 2006.

8. Previdelli AN, Andrade SC, Pires MM, Ferreira SR, Fisberg RM, Marchioni DM. Índice de Qualidade da Dieta Revisado para população brasileira. Rev Saude Publica 2011; 45(4):794-798.

9. Andrade SC, Previdelli AN, Marchioni DM, Fisberg RM. Avaliação da confiabilidade e validade do Índice de Qualidade da Dieta Revisado. Rev Saude Publica 2013; 47(4):675-683.

10. Ford ES, Mokdad AH, Liu S. Healthy Eating Index and $\mathrm{C}$-reactive protein concentration: findings from the National Health and Nutrition Examination Survey III, 1988-1994. Eur J Clin Nutr 2005; 59(2):278-283.

11. De Koning L, Chiuve SE, Fung TT, Willett WC, Rimm EB, Hu FB. Diet-quality scores and the risk of type 2 diabetes in men. Diabetes Care 2011;34(5):1150-1156.

12. Kuczmarski MF, Sees AC, Hotchkiss L, Cotugna N, Evans MK, Zonderman AB. Higher Healthy Eating Index-2005 scores associated with reduced symptoms of depression in an urban population: findings from the Healthy Aging in Neighborhoods of Diversity Across the Life Span (HANDLS) study. J Am Diet Assoc 2010; 110(3):383-389.

13. Chiuve SE, Fung TT, Rimm EB, Hu FB, McCullough ML, Wang M, Stampfer MJ, Willett WC. Alternative Dietary Indices Both Strongly Predict Risk of Chronic Disease. J Nutr 2012; 142(6):1009-1018.

14. Aquino EM, Barreto SM, Bensenor IM, Carvalho MS, Chor D, Duncan BB, Lotufo PA, Mill JG, Molina MDC, Mota ELA, Passos VMAP, Schmidt MI, Szklo M.Brazilian longitudinal study of adult health (ELSA-Brasil): objectives and design. Am J Epidemiol 2012; 175(4):315-324.

15. Molina MD, Faria CP, Cardoso LDO, Drehmer M, Velasquez-Meléndez JG, Gomes ALC, Melere C, Diniz MDFHS, Sichieri R, Benseñor IJM. Diet assessment in the Brazilian Longitudinal Study of Adult Health (ELSA-Brasil): Development of a food frequency questionnaire. Rev Nutr 2013; 26(2):167-176.

16. Center NC. Nutrition Data System for Research (NDS-R). Version. 2008; 4:34. Minneapolis: University of Minnesota; 2008.
17. Instituto Brasileiro de Geografia e Estatística (IBGE). Pesquisa de Orçamentos Familiares 2008-2009: análise do consumo alimentar pessoal no Brasil. Rio de Janeiro: IBGE; 2011.

18. Thompson FE, Subar AF, Smith AF, Midthune D, Radimer KL, Kahle LL, Kipnis V. Fruit and vegetable assessment: performance of 2 new short instruments and a food frequency questionnaire. J Am Diet Assoc 2002; 102(12):1764-1772.

19. Molina MD, Benseñor IM, Cardoso LDO, Velasquez-Melendez G, Drehmer M, Pereira TSS, Faria CP, Melere C, Manato L, Gomes ALC, Fonseca MJM, Sichieri R. Reproducibility and relative validity of the Food Frequency Questionnaire used in the ELSA-Brasil. Cad Saude Publica 2013; 29(2):379-389.

20. Pilger C, Menon, MU, Freitas Mathias, TA. Utilização de serviços de saúde por idosos vivendo na comunidade. Rev Esc Enferm USP 2013; 47(1):213-220.

21. Cardoso LDO, Carvalho MS, Cruz OG, Melere C, Luft VC, Molina, MDCB , Faria CPD, Benseñor IM, Matos SMA, Fonseca MDJMD, Griep RH, Chor D. Eating patterns in the Brazilian Longitudinal Study of Adult Health (ELSA-Brasil): an exploratory analysis. Cad Saude Publica 2016; 32(5):e00066215.

22. Monteiro CA, Conde WL, Castro IR. A tendência cambiante da relação entre escolaridade e risco de obesidade no Brasil (1975-1997). Cad Saude Publica 2003; 19(Supl. 1): S67-S75.

23. Huybrechts I, Vereecken C, De Bacquer D, Vandevijvere S, Oyen HV, Maes L, Vanhauwaert E, Temme L, Backer G, Henauw S.Reproducibility and validity of a diet quality index for children assessed using a FFQ. Br J Nutr 2010; 104(01):135-144.

24. Newby PK, Hu FB, Rimm EB, Smith-Warner SA, Feskanich D, Sampson L, Willett WC. Reproducibility and validity of the Diet Quality Index Revised as assessed by use of a food-frequency questionnaire. Am J Clin Nutr 2003; 78(5):941-949.

25. Brasil. Ministério da Saúde (MS). Guia alimentar para a População Brasileira. Brasília: MS; 2014.

26. Guenther PM, Casavale KO, Reedy J, Kirkpatrick SI, Hiza HAB, Kuczynski KJ, Kahle LL, Krebs-Smith SM. Update of the healthy eating index: HEI-2010. J Acad Nutr Diet 2013; 113(4):569-580.

Artigo apresentado em 05/12/2016 Aprovado em 12/07/2018

Versão final apresentada em 14/07/2018 
